\title{
ABD Matrix of Single-Ply Triaxial Weave Fabric Composites
}

\author{
A.B.H. Kueh* and S. Pellegrino ${ }^{\dagger}$ \\ University of Cambridge, Cambridge, CB2 1PZ, UK
}

\begin{abstract}
The linear-elastic response of single-ply triaxial weave fabric composites is modelled in terms of a homogenized Kirchhoff plate. The ABD matrix for this plate is computed from an assembly of transversely isotropic three-dimensional beams whose unit cell is analysed using standard finite-element analysis, assuming periodic boundary conditions. A subset of the analytical results is validated by means of careful experiments. It is shown that this simple unit cell beam model captures accurately the experimentally observed behaviour.
\end{abstract}

\section{Introduction}

Triaxial weave fabric (TWF) composites are of great interest for future lightweight structures, both rigid and deployable. The fabric is made up of tows woven in three directions, at 0 degrees and \pm 60 degrees; it is impregnated with resin and cured in an autoclave, like a standard composite. A particular attraction of this material is that it is mechanically quasi-isotropic, on a macroscopic scale, and hence can be used to construct single-ply structural elements of very low areal mass. Figure 1 shows a photograph of two spacecraft reflectors made from TWF. Note that one can "see through" these structure, due to the high degree of porosity of the material.

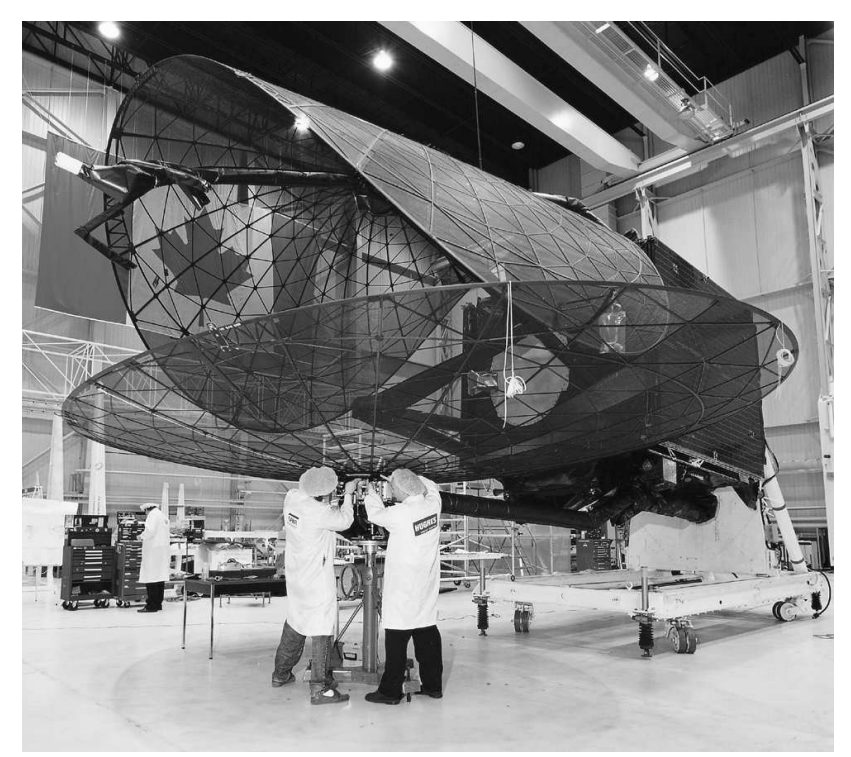

Figure 1. Spring back reflectors (one folded and one deployed) on MSAT-2 spacecraft, from reference 1.

The behaviour of this material is more subtle than standard composite laminates, as in single-ply woven fabrics some of the three-dimensional degrees of freedom remain unconstrained. This results in some

\footnotetext{
* Research student, Department of Engineering, Trumpington Street.

${ }^{\dagger}$ Professor of Structural Engineering, Department of Engineering, Trumpington Street. Associate Fellow AIAA.
} 
important differences between the behaviour of single-ply TWF composites and standard laminates, which include:

- three-dimensional behaviour, leading to coupling between in-plane and out-of-plane effects;

- thermally-induced twist;

- geometrically non-linear variation of in-plane stiffnesses, as the TWF becomes stiffer at larger strains, due to the straightening of the tows;

- variation of the Poisson's ratio.

These effects have been recently investigated ${ }^{2-4}$ but there are still a number of open issues, both in terms of modelling techniques and the experimental verification of the numerical models.

In this paper we focus on the mechanical response of single-ply TWF to small strains, thus neglecting any geometrically non-linear or thermo-elastic effects. We introduce a homogenized Kirchhoff plate model where the generalised strains are the three mid-plane strains and the three mid-plane curvatures, and the corresponding stress resultants are the mid-plane forces and out-of-plane bending and twisting moments per unit length. In analogy with standard laminated composites, the $6 \times 6$ constitutive matrix is denoted as ABD. This matrix is computed by modelling the TWF composite as an assembly of transversely isotropic three-dimensional beams whose unit cell is analysed using standard finite-element analysis, by assuming periodic boundary conditions. A subset of the analytical results is validated by means of carefully designed experiments.

Our aims are: (i) to establish the limitations of a simple unit-cell approach that does not include additional variables, as it has clear advantages in terms of practical applications; (ii) to test the accuracy of the ABD matrix obtained from a beam model, as opposed to the solid model considered by Aoki and Yoshida. ${ }^{2}$ We show that the simple unit cell beam model is able to capture accurately the experimentally observed behaviour of single-ply TWF.

The paper is arranged as follows. In section II we describe the carbon fibre TWF composite that we have studied; estimates for the mechanical properties of a single tow are obtained. In section III we introduce our modelling technique and provide details for carrying out the calculations with the finite-element package ABAQUS. In sections IV to VII we present four sets of tests in which we have measured the initial stiffnesses in tension/compression, shear and bending; the failure load in each mode was also measured. In section VIII we compare experimental and theoretical results. Section IX concludes the paper.

\section{Material Description}

A representative unit cell of TWF is shown in figure 2. The particular type of TWF that is studied in this paper is SK-802, produced by Sakase-Adtech Ltd., Japan. This fabric consists of 1000 filaments of T300 carbon fibre, produced by Toray Industries Inc., Japan, woven in the "basic weave" pattern. For the matrix, we use the resin Hexcel 8552, from Hexcel Composites, UK. In the basic weave pattern the hexagonal holes cover about half of the area. SK-802 has a dry mass of $75 \mathrm{~g} / \mathrm{m}^{2}$ and a thickness of about $0.15 \mathrm{~mm}$. The properties of the two constituents, provided by the suppliers, ${ }^{5-7}$ are listed in table 1 . We define the $x$-direction of the weave to be aligned with the direction of the 0 degree tows.

Table 1. Fibre and matrix material properties

\begin{tabular}{lcc}
\hline \hline Properties & T300 fibre & Hexcel 8552 matrix \\
\hline Density, $\rho\left[\mathrm{kg} / \mathrm{m}^{3}\right]$ & 1,760 & 1,301 \\
Longitudinal stiffness, $E_{1}\left[\mathrm{~N} / \mathrm{mm}^{2}\right]$ & 233,000 & 4,760 \\
Transverse stiffness, $E_{2}\left[\mathrm{~N} / \mathrm{mm}^{2}\right]$ & 23,100 & 4,760 \\
Shear stiffness, $G_{12}\left[\mathrm{~N} / \mathrm{mm}^{2}\right]$ & 8,963 & 1,704 \\
Poisson's ratio, $\nu_{12}$ & 0.2 & 0.37 \\
Maximum strain, $\varepsilon_{\max }[\%]$ & 1.5 & 1.7 \\
\hline
\end{tabular}




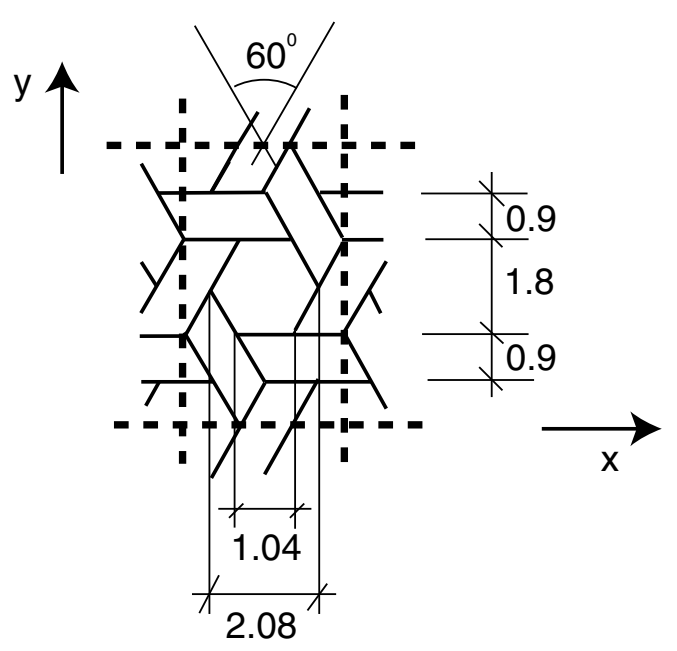

Figure 2. Dimensions, in $\mathrm{mm}$, of SK-802 unit cell

The material is processed as follows. The fabric is laid on release paper and one layer of $30 \mathrm{~g} / \mathrm{m}^{2} \mathrm{semi}$ solid resin film, for a single side infusion, is placed on the fabric. It is the vacuum-bagged in a standard way, and the lay-up is first heated to a temperature of $110^{\circ} \mathrm{C}$ and a pressure of 6 bar for 1 hour in an autoclave, allowing the resin to flow and spread evenly through the fibres. They are then cured at a temperature of $185^{\circ} \mathrm{C}$ with the same pressure, for 2 hours.

\section{A. Tow Properties}

Figure 3 is a micrograph showing the tow cross-sections. The exact shape and orientation vary slightly, depending on where the section is taken, but in general the cross-sections consist of a central part, roughly of constant height, tapering to a point on either side. The average tow width, measured from a series of micrographs, is $0.85 \mathrm{~mm}$. The average tow cross-sectional area is $0.0626 \mathrm{~mm}^{2}$.

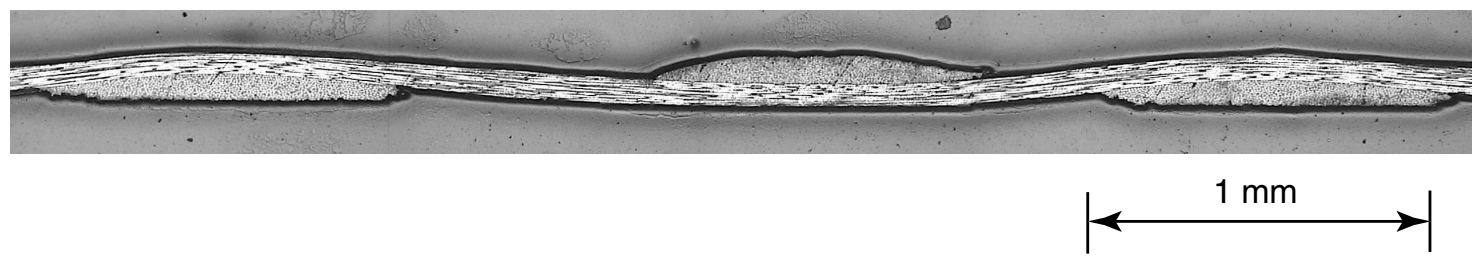

Figure 3. Micrograph of tow cross sections

Each tow will be modelled as a beam element, whose engineering constants are determined as follows..$^{3,4,8}$ The subscripts $f$ and $m$ denote fibre and matrix, respectively.

The longitudinal extensional modulus and the Poisson's ratio are obtained from the rule of mixtures

$$
\begin{gathered}
E_{1}=E_{1 f} V_{f}+E_{m}\left(1-V_{f}\right) \\
\nu_{12}=\nu_{13}=\nu_{12 f} V_{f}+\nu_{m}\left(1-V_{f}\right)
\end{gathered}
$$

The transverse extensional modulus is found from the Halpin-Tsai semi-empirical relation ${ }^{8}$

$$
E_{2}=E_{3}=E_{m} \frac{1+\xi \eta V_{f}}{1-\eta V_{f}}
$$


where

$$
\eta=\frac{E_{2 f}-E_{m}}{E_{2 f}+\xi E_{m}}
$$

and the parameter $\xi$ is a measure of reinforcement of the composite that depends on the fibre geometry, packing geometry, and loading conditions. It has been set equal to 2.0.

The shear modulus $G_{12}=G_{13}$ is found from the Halpin-Tsai semi-empirical relation

$$
G_{12}=G_{13}=G_{m} \frac{\left(G_{12 f}+G_{m}\right)+V_{f}\left(G_{12 f}-G_{m}\right)}{\left(G_{12 f}+G_{m}\right)-V_{f}\left(G_{12 f}-G_{m}\right)}
$$

The computation of the in-plane shear modulus, $G_{23}$, is done according to reference 9 (it is quite lengthy and hence the details are not shown here). Subsequently, $\nu_{23}$ is computed from

$$
G_{23}=\frac{E_{2}}{2\left(1+\nu_{23}\right)}
$$

Using the measured volume fraction, $V_{f}=0.67$, the engineering constants used to define the beam element are listed in table 2. Note that the longitudinal stiffness of the beams is much greater than the transverse stiffnesses.

Table 2. T300/Hexcel 8552 tow material properties

\begin{tabular}{lc}
\hline \hline Material Properties & Value \\
\hline Longitudinal stiffness, $E_{1}\left[\mathrm{~N} / \mathrm{mm}^{2}\right]$ & 157,650 \\
Transverse stiffness, $E_{2}=E_{3}\left[\mathrm{~N} / \mathrm{mm}^{2}\right]$ & 13,280 \\
Shear stiffness, $G_{12}=G_{13}\left[\mathrm{~N} / \mathrm{mm}^{2}\right]$ & 4,561 \\
In-plane shear stiffness, $G_{23}\left[\mathrm{~N} / \mathrm{mm}^{2}\right]$ & 4,538 \\
Poisson's ratio, $\nu_{12}=\nu_{13}$ & 0.256 \\
\hline \hline
\end{tabular}

\section{Homogenization of Unit Cell}

\section{A. Model of Unit Cell}

Figure 4 shows a perspective view of the unit cell defined in Figure 2, where each tow is represented by its centre line. Note that at the cross-over points the centre line offset is equal to the thickness of one tow. Also note that it is assumed that the connection between the tow centre lines can be modelled as fully rigid.

Our simulation, carried out with the finite element package ABAQUS Version 6.4, uses a transversely isotropic, geometrically linear model of the beam, modelled with the three-dimensional 3-node quadratic beam element, B32. This element is derived from Timoshenko beam theory and so allows for transverse shear deformation. The complete model for a unit cell consists of 494 nodes and 248 beam elements.

A uniform rectangular beam cross section area of $0.803 \mathrm{~mm}$ wide $\times 0.078 \mathrm{~mm}$ thick is assumed. The beam cross-over points are connected using the multi-point-constraint * MPC function in ABAQUS. *MPC type *BEAM is chosen to represent the connection between two crossing beams. *BEAM provides a full, rigid connection between the centre-line points that are directly above one another.

There are 8 boundary nodes for this unit cell, all in the cell mid-plane; one on the top and bottom edges and three on the right and left edges (labelled $i$, ii, and $i i i$ in the figure). Note that the nodes labelled $i$ and iii, on the right and left edges, coincide with the end nodes of the beams that model the 0-direction tows; the other four nodes are located half-way between the cross-over points.

\section{B. ABD Matrix}

We represent the TWF unit cell in terms of a thin Kirchhoff plate. Hence our kinematic variables are the mid-plane strains $\varepsilon_{x}=\partial u / \partial x, \varepsilon_{y}=\partial v / \partial y, \varepsilon_{x y}=(\partial u / \partial y)+(\partial v / \partial x)$, where it should be noted that the engineering shear strain is used, and $\kappa_{x}=-\partial^{2} w / \partial x^{2}, \kappa_{y}=-\partial^{2} w / \partial y^{2}, \kappa_{x y}=-2 \partial^{2} w / \partial x \partial y$, where it 


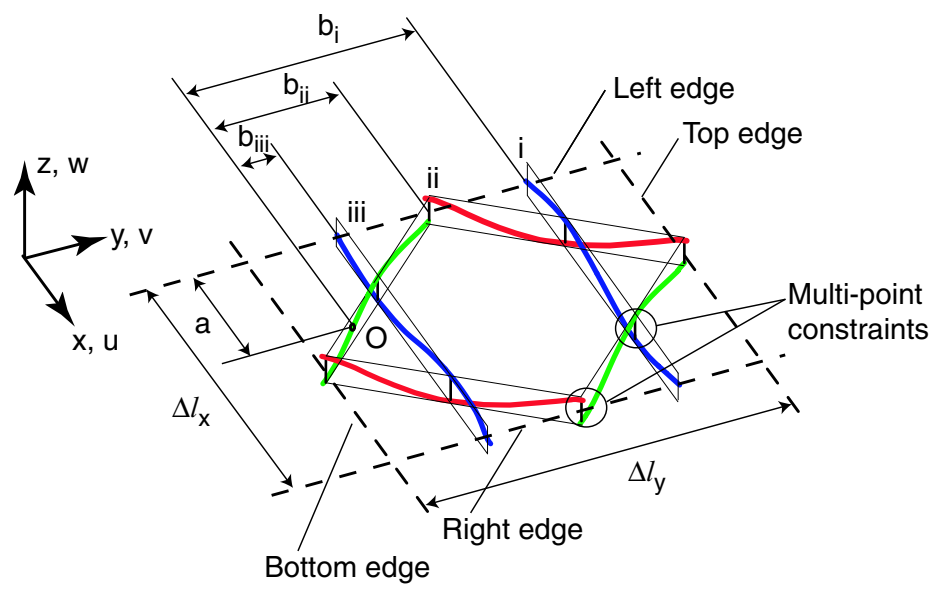

(a)

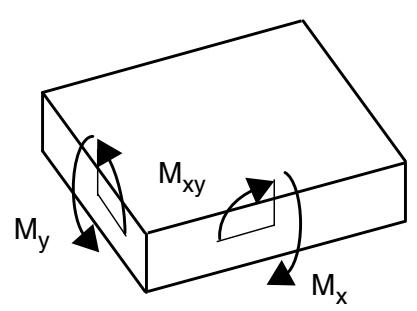

(b)

Figure 4. (a) Perspective view of TWF unit cell; (b) moment sign convention for plate.

should be noted that twice the surface twist is used, as it is standard in the theory of laminated plates. ${ }^{8}$ The corresponding static variables are the mid-plane force and moment resultants per unit length $N_{x}, N_{y}, N_{x y}$ and $M_{x}, M_{y}, M_{x y}$.

In analogy with classical laminate theory we write the $6 \times 6$ matrix relating the two sets of variables as an ABD stiffness matrix,

$$
\left\{\begin{array}{c}
N_{x} \\
N_{y} \\
N_{x y} \\
M_{x} \\
M_{y} \\
M_{x y}
\end{array}\right\}=\left[\begin{array}{ccccccc}
A_{11} & A_{12} & A_{16} & \mid & B_{11} & B_{12} & B_{16} \\
A_{21} & A_{22} & A_{26} & \mid & B_{21} & B_{22} & B_{26} \\
A_{61} & A_{62} & A_{66} & \mid & B_{61} & B_{62} & B_{66} \\
\hline B_{11} & B_{21} & B_{61} & \mid & D_{11} & D_{12} & D_{16} \\
B_{12} & B_{22} & B_{62} & \mid & D_{21} & D_{22} & D_{26} \\
B_{16} & B_{26} & B_{66} & \mid & D_{61} & D_{62} & D_{66}
\end{array}\right]\left\{\begin{array}{c}
\varepsilon_{x} \\
\varepsilon_{y} \\
\varepsilon_{x y} \\
-\kappa_{x} \\
\kappa_{y} \\
\kappa_{x y}
\end{array}\right\}
$$

This matrix is symmetric, and so the $3 \times 3$ submatrices $\mathrm{A}$ and $\mathrm{D}$ along the main diagonal are symmetric $\left(A_{i j}=A_{j i}\right.$ and $D_{i j}=D_{j i}$ ), however (unlike the B matrix of a laminate) the B matrix is not guaranteed to be symmetric.

\section{Periodic Boundary Conditions}

Periodic boundary conditions are a standard tool in the computation of homogenized models for composites and so there is an extensive literature on this topic. A recent paper by Tang and Whitcomb ${ }^{10}$ explains the key ideas involved in this approach, in the context of semi-analytical solutions based on assumed displacement fields within the unit cell. Of particular relevance to the present study is the direct micro-mechanics method introduced by Karkkainen and Sankar ${ }^{11}$ for plain weave textile composites. The approach presented here is essentially that of this reference, but (i) extended to a triaxial weave and (ii) discretizing the TWF into a mesh of beam elements, instead of solid elements.

Consider a homogenized plate subject to uniform mid-plane strains $\bar{\varepsilon}_{i j}$ and uniform mid-plane curvatures $\bar{\kappa}_{i j}$. The periodic boundary conditions, for the changes in a displacement component, $\Delta u_{i}$, and rotation component, $\Delta \theta_{i}$, of corresponding nodes on opposite boundaries, can be defined as follows,

$$
\begin{aligned}
& \Delta u_{i}=\bar{\varepsilon}_{i j} \Delta l_{j} \\
& \Delta \theta_{i}=\bar{\kappa}_{i j} \Delta l_{j}
\end{aligned}
$$


where $\Delta l_{j}$ is the distance in the $u$-direction between two corresponding nodes.

Note that we use the subscripts R, L, T, and B to denote nodes on the right, left, top, and bottom edges of the unit cell, respectively, and $i, i i$, and $i i i$ to distinguish between different side nodes. Also note that only stretching/shearing and out-of-plane bending/twisting of the mid-plane are considered. In-plane bending and shearing are not considered.

To prevent a rigid body motion, one point on the unit cell, point $\mathrm{O}$ in figure 4(a), has been constrained against translation in any direction.

The PBC of two corresponding nodes are set as follows. For the left/right edges,

$$
\begin{aligned}
u_{R n}-u_{L n} & =\bar{\varepsilon}_{x x} \Delta l_{x} \\
v_{R n}-v_{L n} & =\bar{\varepsilon}_{x y} \Delta l_{x} / 2 \\
w_{R n}-w_{L n} & =\bar{\kappa}_{x y} b \Delta l_{x} / 2 \\
\theta_{R x n}-\theta_{L x n} & =-\bar{\kappa}_{x y} \Delta l_{x} / 2 \\
\theta_{R y n}-\theta_{L y n} & =\bar{\kappa}_{x x} \Delta l_{x} \\
\theta_{R z n}-\theta_{L z n} & =0
\end{aligned}
$$

where

$$
\begin{array}{ll}
n & =i, i i, i i i \text { sets of nodes on left/right boundaries } \\
R, L & =\text { right and left boundary, respectively } \\
x, y, \text { and } z & =\text { directions of cartesian coordinate system }
\end{array}
$$

For the top/bottom edges,

$$
\begin{aligned}
u_{T}-u_{B} & =\bar{\varepsilon}_{x y} \Delta l_{y} / 2 \\
v_{T}-v_{B} & =\bar{\varepsilon}_{y y} \Delta l_{y} \\
w_{T}-w_{B} & =\bar{\kappa}_{x y} a \Delta l_{y} / 2 \\
\theta_{T x}-\theta_{B x} & =\bar{\kappa}_{y y} \Delta l_{y} \\
\theta_{T y}-\theta_{B y} & =-\bar{\kappa}_{x y} \Delta l_{y} / 2 \\
\theta_{T z}-\theta_{B z} & =0
\end{aligned}
$$

In ABAQUS, we simulate these PBCs using the *EQUATION and *BOUNDARY commands. Dummy nodes are used to represent the deformation terms, strain or curvature, in equations (10) to (21). The constraint forces and moments that correspond to each dummy node action are then used to compute the $\mathrm{ABD}$ matrix for the composite, as explained in the next section.

\section{Virtual Work Calculation of ABD Matrix}

Six unit deformations are imposed on the unit cell, in six separate ABAQUS analyses. In each case we set one average strain/curvature equal to one and all others equal to zero. For instance, in the first analysis, $\bar{\varepsilon}_{x x}=1$ while $\bar{\varepsilon}_{y y}=\bar{\varepsilon}_{x y}=0$ and $\bar{\kappa}_{x x}=\bar{\kappa}_{y y}=\bar{\kappa}_{x y}=0$. From each of the six analyses we obtain one set of deformations, including displacement and rotation components at the 8 boundary nodes, and one set of corresponding constraint forces and moments.

Next, we use virtual work to compute the entries of the ABD matrix. For example, entry 1,1 is obtained by writing the equation of virtual work for the first deformation mode (i.e. $\bar{\varepsilon}_{x x}=1$ ) and the forces/moments also in the first mode. Hence the equation reads

$$
N_{x x} \bar{\varepsilon}_{x x} \Delta l_{x} \Delta l_{y}=\sum_{b . n .}\left(F_{x} u+F_{y} v+F_{z} w+M_{x} \theta_{x}+M_{y} \theta_{y}+M_{z} \theta_{z}\right)
$$

where the summation is extended to the 8 boundary nodes (b.n.). Then, substituting $\bar{\varepsilon}_{x x}=1$ and comparing with equation (7) we obtain

$$
A_{11}=\frac{\sum_{b . n .}\left(F_{x} u+F_{y} v+F_{z} w+M_{x} \theta_{x}+M_{y} \theta_{y}+M_{z} \theta_{z}\right)}{\Delta l_{x} \Delta l_{y}}
$$


The calculation of the whole ABD matrix is best done by setting up two matrices with 48 rows (i.e. 6 degrees of freedom per node times 8 boundary nodes) and 6 columns (i.e. the six deformation modes). The first matrix, $U$, contains in each column the displacement and rotation components at all boundary nodes for one deformation mode; the second matrix, $F$, contains in each column the forces and moments at all boundary nodes for one deformation mode. The ABD matrix is then given by

$$
A B D=\frac{U^{T} F}{\Delta l_{x} \cdot \Delta l_{y}}
$$

The outcome of this calculation is

$$
\left\{\begin{array}{c}
N_{x} \\
N_{y} \\
N_{x y} \\
M_{x} \\
M_{y} \\
M_{x y}
\end{array}\right\}=\left[\begin{array}{ccccccc}
3411.10 & 2050.20 & 0 & \mid & 0 & 0 & -0.64 \\
2050.20 & 3411.00 & 0 & \mid & 0 & 0 & 0.64 \\
0 & 0 & 680.42 & \mid & 0.64 & -0.64 & 0 \\
0 & 0 & 0.64 & \mid & 2.17 & 0.60 & 0 \\
0 & 0 & -0.64 & \mid & 0.60 & 2.17 & 0 \\
-0.64 & 0.64 & 0 & \mid & 0 & 0 & 0.78
\end{array}\right]\left\{\begin{array}{c}
\varepsilon_{x} \\
\varepsilon_{y} \\
\varepsilon_{x y} \\
\hline \kappa_{x} \\
\kappa_{y} \\
\kappa_{x y}
\end{array}\right\}
$$

where the units are $\mathrm{N}$ and $\mathrm{mm}$.

Aoki ad Yoshida ${ }^{2}$ have argued that, because TWF is quasi-isotropic, both the A and D matrices have to satisfy the conditions met by an isotropic plate, namely $A_{11}=A_{22}, A_{66}=\left(A_{11}-A_{12}\right) / 2$ and $D_{11}=$ $D_{22}, D_{66}=\left(D_{11}-D_{12}\right) / 2$. It can be verified that all of these conditions are satisfied by the matrix in equation (25).

\section{Tension Tests}

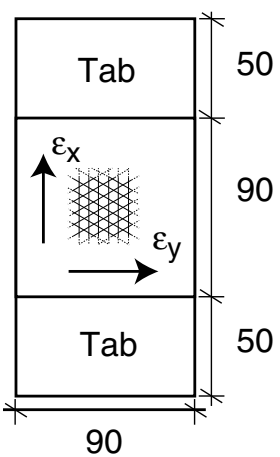

(a)

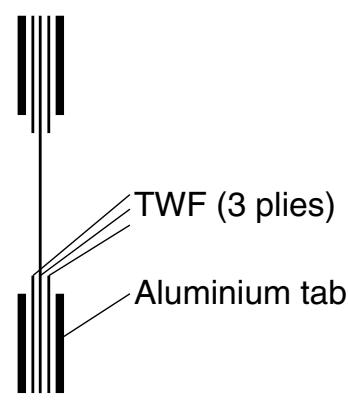

(b)

Figure 5. Specimen used in the tension tests (dimensions in $\mathrm{mm}$ ); (a) front view; (b) edge view

\section{A. Specimen and Testing Procedure}

The layout of the tensile test specimen is shown in figure $5 .{ }^{12}$ To minimize edge effects, we have adopted an aspect ratio of $1: 1$; the $90 \mathrm{~mm}$ wide specimens include 16 unit cells. The specimens were cut with straight edges and sandwiched at each end between two additional $60 \mathrm{~mm} \times 90 \mathrm{~mm}$ TWF layers and two $90 \mathrm{~mm}$ by $50 \mathrm{~mm}$ aluminium tabs, $1 \mathrm{~mm}$ thick. These additional reinforcements serve the purpose of preventing premature failure occurring near the clamped areas. The extra TWF layers were glued to the centre layer with Araldite epoxy resin mixed with hardener at a ratio of 1:1. The aluminum tabs were attached to the specimen's ends with an industrial superglue. 
(a)

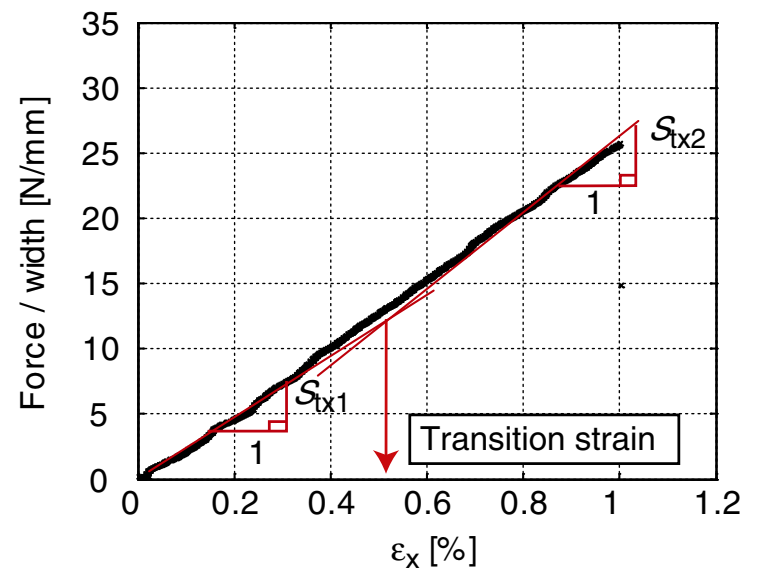

(b)

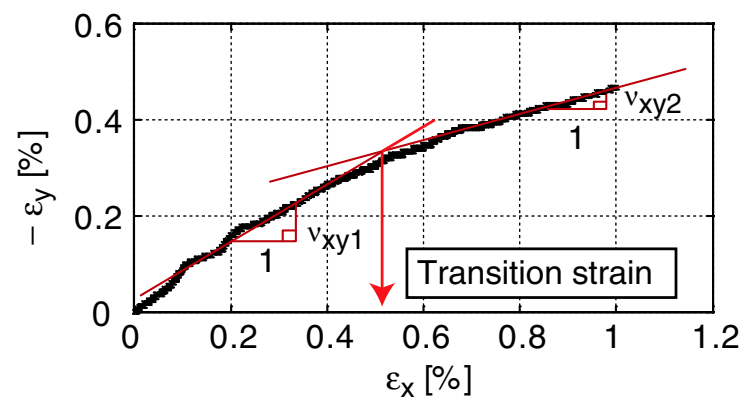

Figure 6. Definition of transition strain

Ten specimens were tested. Strain measurements were carried out with Epsilon LE-01 and LE-05 laser extensometers, using two pairs of $3 \mathrm{~mm}$ wide retro-reflective strips attached to the central region of the specimen, about $50 \mathrm{~mm}$ apart each in the longitudinal and transverse direction, on the specimen center. The specimens were gripped over the aluminium tabs between two wedged clamping jaws. The tests were carried out with an Instron 5578 testing machine fitted with a $30 \mathrm{KN}$ load cell, following the procedure in reference 12 . An extension rate of $1 \mathrm{~mm} / \mathrm{min}$ was used.

\section{B. Results}

Figure 6(a) shows a plot of longitudinal force per unit width vs longitudinal strain for specimen T6. The load-deformation plot is mildly non-linear. Following reference 3 we approximate the response curve with two straight lines; the response at smaller strains is that of a grillage of "wavy-tows", whereas at larger strains the tows along the main load-bearing path are essentially straight. The slopes of these lines define the small-strain (subscript 1) tensile stiffness of the specimen, $S_{t x 1}$ and the large-strain stiffness, $S_{t x 2}$. In the plot, the intersection between the two straight lines defines the transition strain.

A non-linearity is also observed in the transverse strain-longitudinal strain plot, figure 6(b). We fit two straight lines to this curve ${ }^{3}$ and hence define two Poisson's ratios, $\nu_{x y 1}$ and $\nu_{x y 2}$; the subscript $t$ has been dropped because the Poisson's ratio in compression will not be measured and so there is no need to distinguish between tension and compression.

The results for the full set of tests are summarised in figure 7 and figure 8 . Table 3 presents both stiffness and Poisson's ratio, before and after the transition strain. The failure stress, $\sigma_{t}^{u}$, and strain, $\varepsilon_{t}^{u}$, are also shown. Note that in all cases the stiffness increases and the Poisson's ratio decreases, once the transition strain is reached. 


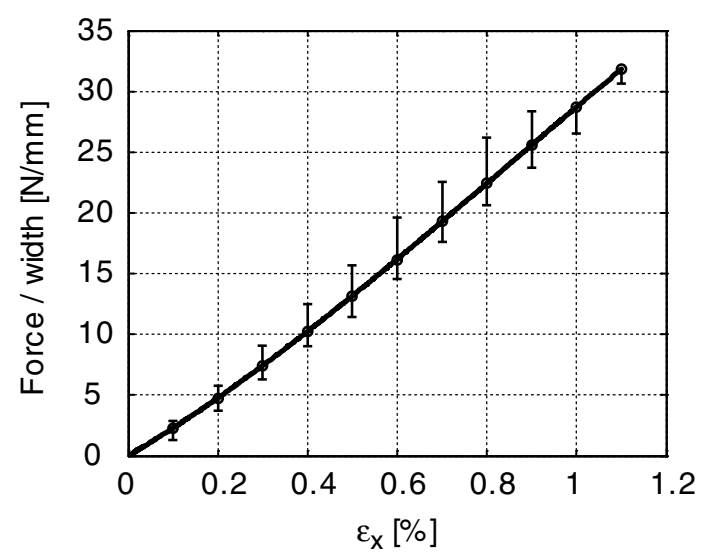

Figure 7. Measured force per unit width vs strain response from tension test

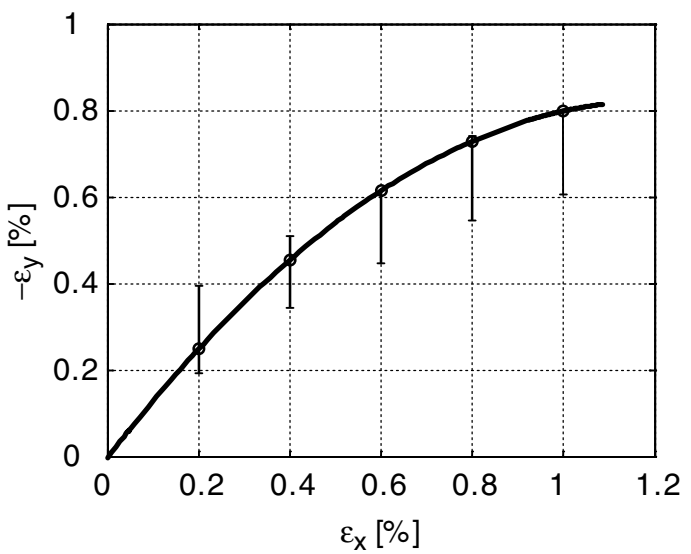

Figure 8. Measured transverse strain vs longitudinal strain response from tension test

Table 3. Tension test results

\begin{tabular}{ccccccc}
\hline \hline Specimen & $\begin{array}{c}S_{t x 1} \\
{[\mathrm{~N} / \mathrm{mm}]}\end{array}$ & $\begin{array}{c}S_{t x 2} \\
{[\mathrm{~N} / \mathrm{mm}]}\end{array}$ & $\nu_{x y 1}$ & $\nu_{x y 2}$ & $\begin{array}{c}\sigma_{t}^{u} \\
{[\mathrm{MPa}]}\end{array}$ & $\begin{array}{c}\varepsilon_{t}^{u} \\
{[\%]}\end{array}$ \\
\hline T1 & 2100.3 & 3278.7 & 0.564 & 0.428 & 198.53 & 1.11 \\
T2 & 2062.8 & 3015.1 & 0.541 & 0.360 & 160.19 & 0.90 \\
T3 & 2067.0 & 3028.0 & 0.663 & 0.422 & 165.58 & 0.91 \\
T4 & 2069.5 & 3129.7 & 0.561 & 0.408 & 163.10 & 0.93 \\
T5 & 2245.8 & 3088.0 & 0.658 & 0.466 & 139.42 & 0.81 \\
T6 & 2250.0 & 3075.6 & 0.564 & 0.358 & 164.91 & 1.00 \\
T7 & 2306.9 & 3116.1 & 0.542 & 0.353 & 191.28 & 1.00 \\
T8 & 2261.2 & 3172.9 & 0.520 & 0.366 & 183.91 & 0.98 \\
T9 & 2069.5 & 3066.5 & 0.558 & 0.421 & 150.38 & 0.84 \\
T10 & 2024.8 & 3098.0 & 0.690 & 0.388 & 183.46 & 1.08 \\
\hline Average & 2145.8 & 3106.9 & 0.586 & 0.389 & 170.08 & 0.96 \\
Std. dev. & 106.239 & 76.141 & 0.060 & 0.038 & 18.709 & 0.097 \\
Variation [\%] & 4.95 & 2.45 & 10.27 & 9.73 & 11.00 & 10.12 \\
\hline \hline
\end{tabular}




\section{Compression Tests}

Compression tests on thin composites are notoriously difficult, as the failure mode of interest is fibre microbuckling but other test-dependent failure modes tend to occur at lower stress. Following reference 13, we carry out the compression tests on short sandwich columns, as shown in figure 9 . This reference shows that by a suitable choice of the properties of the foam the lateral restraint provided by the core can be optimized to prevent failure by overall Euler buckling, core shear macrobuckling, and face wrinkling so that the specimen fails by fibre microbuckling.

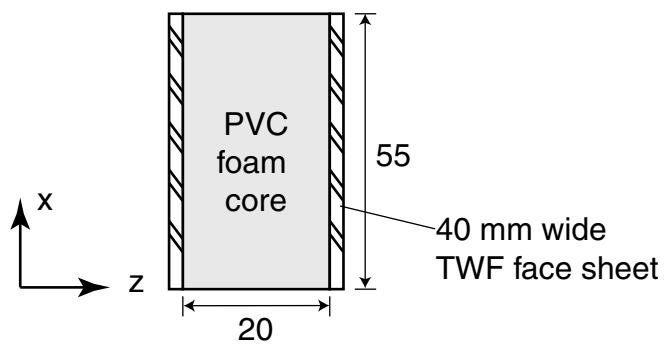

Figure 9. Specimen for compression test (dimensions in $\mathbf{m m}$ )

\section{A. Specimen and Test Procedure}

To minimize edge effects, nearly-square TWF sheets, of size $40 \mathrm{~mm}$ by $55 \mathrm{~mm}$, were tested. These sheets were bonded to a $20 \mathrm{~mm}$ thick sandwich core of closed-cell Polyvinyl chloride (PVC) foam (trade name: Divinycell, density $186 \mathrm{~kg} / \mathrm{m}^{3}$ ). The TWF face sheets were bonded to the PVC core using Araldite resin, mixed with hardener at a ratio of 1:1.

Ten nominally identical specimens were tested with an Instron 5578 testing machine, fitted with a $2 \mathrm{KN}$ load cell. The load was applied via flat steel platens. A loading rate of $1 \mathrm{~mm} / \mathrm{min}$ was applied. The longitudinal deformation on both surfaces of the specimen was measured with Epsilon LE-01 and LE-05 laser extensometers, using pairs of retro-reflective strips placed about $30 \mathrm{~mm}$ apart.

\section{B. Results}

The results of the compression tests are summarised in figure 10, and key results are listed in table 4 . The behaviour of TWF in compression is non-linear, with failure occurring at strains in the range $0.41 \%$ to $0.77 \%$ (note the large spread in these values). The scatter in the compressive stiffness, $S_{c x}$, is much smaller.

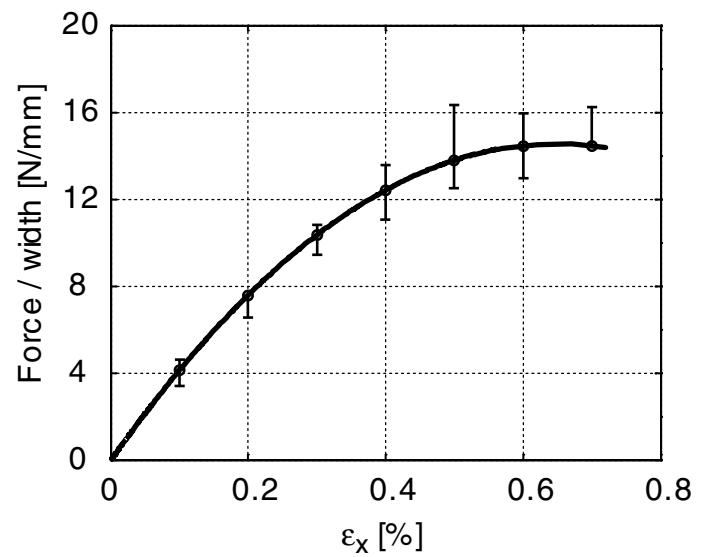

Figure 10. Measured compressive force/width vs strain curve 


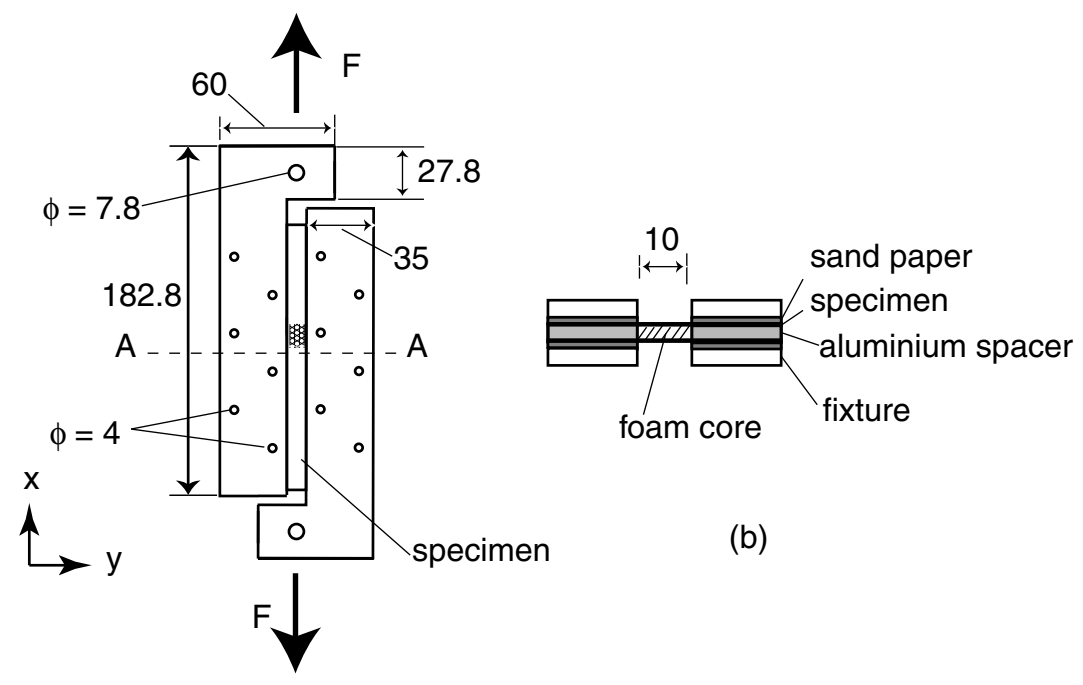

(a)

Figure 11. (a) Modified two rail shear rig; (b) enlarged section A-A (dimensions in mm)

Table 4. Compression test results

\begin{tabular}{cccc}
\hline \hline Specimen & $S_{c x}[\mathrm{~N} / \mathrm{mm}]$ & $\sigma_{c}^{u}[\mathrm{MPa}]$ & $\varepsilon_{c}^{u}[\%]$ \\
\hline C01 & 2178.8 & 110.10 & 0.52 \\
C02 & 2218.7 & 108.49 & 0.72 \\
C03 & 2220.9 & 100.85 & 0.52 \\
C04 & 2290.3 & 95.92 & 0.57 \\
C05 & 2299.4 & 87.80 & 0.77 \\
C06 & 2280.5 & 100.69 & 0.57 \\
C07 & 2346.2 & 110.60 & 0.60 \\
C08 & 2328.7 & 87.25 & 0.64 \\
C09 & 2247.1 & 108.65 & 0.41 \\
C10 & 2036.9 & 79.81 & 0.62 \\
\hline Average & 2244.75 & 99.02 & 0.59 \\
Std. dev. & 89.742 & 10.997 & 0.103 \\
Variation [\%] & 4.00 & 11.11 & 17.36 \\
\hline \hline
\end{tabular}

\section{In-plane Shear Tests}

These tests aim to determine the in-plane shear properties of TWF. We used a modified version of the standard two-rail shear test method, ${ }^{14}$ see figure 11, as this fixture accommodates wide specimens and hence minimizes edge effects. In view of a comment by Adams, Carlsson and Pipes ${ }^{15}$ a 90 degree test fixture was used, instead of the 97 degree fixture in reference 14. Further modifications include the increase in the number of bolt holes from three to six smaller holes per rail, as large holes in TWF may result in high stress concentrations. In addition, sheets of sandpaper were used at the interface, in the clamped region, for a better grip of the specimen.

A preliminary test on a single-ply TWF specimen showed local buckling around the top and bottom free edges of the specimen right from the start, followed by the gradual development of extensive waviness over 
the whole unsupported region of the specimen. Due to the thinness of the material $(t=0.156 \mathrm{~mm})$, it is impossible to avoid these effects in a single sheet configuration and, since the data measured in a buckled state would not be representative of the material shear properties, we decided to use sandwich specimens similar to those used in the compression tests.

\section{A. Specimens and Testing Procedure}

The specimens were $80 \mathrm{~mm}$ wide by $130 \mathrm{~mm}$ long, with an unsupported width of $10 \mathrm{~mm}$. Two TWF sheets were bonded with Evo-stik impact adhesive which sets in 15 - 30 minutes to a $3 \mathrm{~mm}$ thick PVC foam core of the same type as that used in the compression tests, see section V.A. An initial guess of $3 \mathrm{~mm}$ for the foam thickness proved adequate.

Five shear tests were carried out with an Instron 5578 testing machine, mounted with a $2 \mathrm{KN}$ load cell. A loading rate of $0.5 \mathrm{~mm} / \mathrm{min}$ was used.

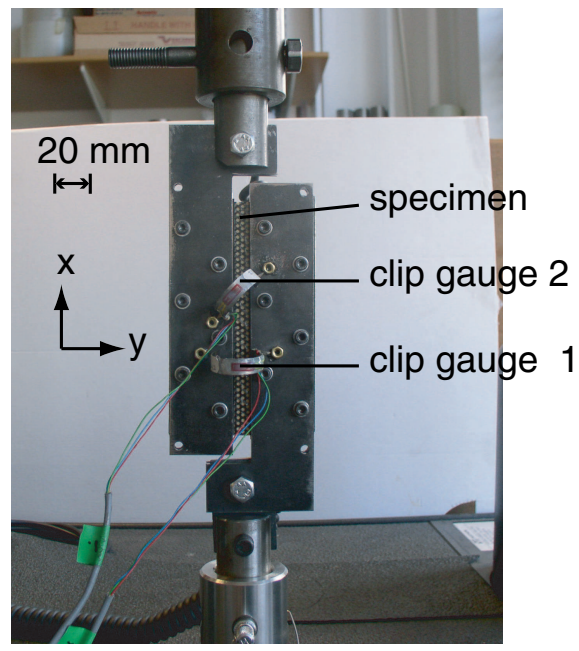

Figure 12. Shear test set up with clip gauges

Measuring the shear strain proved quite challenging. Routinely strain gauges are used, however these cannot be bonded to TWF. An initial attempt was to use the laser extensometers with retro-reflective strips mounted on the unsupported region of the specimens. However, the specimen rotates under load and so two extensometers are insufficient to determine the shear strain. Next, a photogrammetry method was attempted but proved to be too noisy. The targets used were $0.5 \mathrm{~mm}$ long rods of white rubber with a diameter of $0.6 \mathrm{~mm}$, glued to the TWF tows using polyvinyl acetate (PVA) adhesive. A 10.0 megapixel digital camera was used to capture images of a $10 \mathrm{~mm}$ wide region during the test, then the $x$ and $y$ coordinates of the centroid of each target were measured using the sub-pixel resolution function in PhotoModeler Pro 5.2.3. The specimen shear strain was then computed. The resulting strain measurements, see figure 13, are very noisy.

The measurement technique that was finally chosen uses two clip gauges attached to the steel test fixtures, one across the specimen and one at 45 degrees, see figure 12. A clip gauge is a round strip of very thin spring steel fitted with surface mounted strain gauges that measure the curvature of the strip. The tips of the clip tips fit into small holes punched on nuts attached to the test fixture. During the test, each clip gauge measures the distance between two nuts, from which the corresponding strains are computed at the end of the test. Then, the shear strain is computed from

$$
\varepsilon_{x y}=2 \varepsilon_{2}-\varepsilon_{1}
$$

where the subscripts 1 and 2 correspond to the clip gauge number. Note that we the strain parallel to the fixture is neglected. 


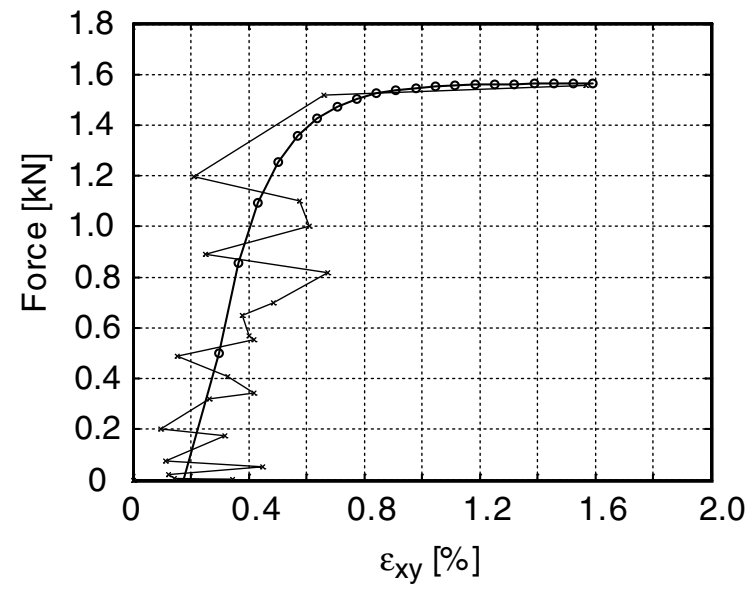

Figure 13. Measured shear force vs shear strain using photogrammetry

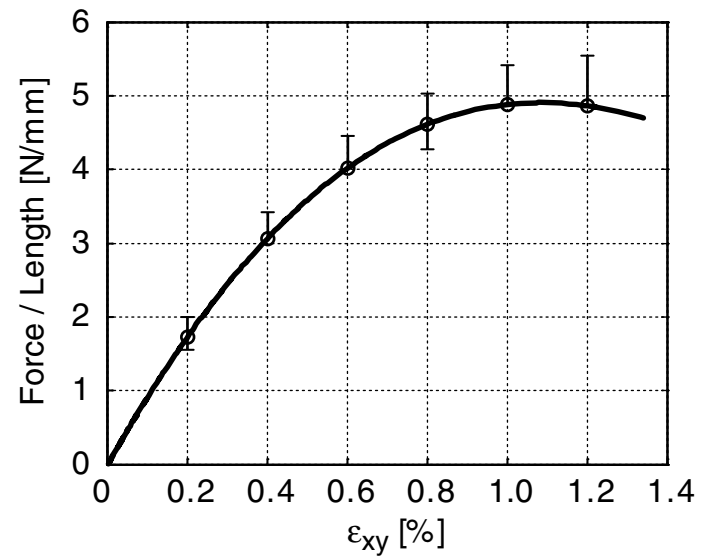

Figure 14. Shear force per unit length vs shear strain 


\section{B. Results}

The relationship between shear force per unit length vs shear strain, obtained from the clip gauge readings, is shown in figure 14. The measured shear properties are then summarized in table 5 . The shear failure stress and strain are represented by $\sigma_{s}^{u}$ and $\varepsilon_{x y}^{u}$, respectively. No failures originating from the edge were observed in the tests that were carried out.

Table 5. Shear test results

\begin{tabular}{cccc}
\hline \hline Specimen & $S_{x y}[\mathrm{~N} / \mathrm{mm}]$ & $\sigma_{s}^{u}[\mathrm{MPa}]$ & $\varepsilon_{x y}^{u}[\%]$ \\
\hline $\mathrm{SC} 1$ & 666.0 & 28.05 & 0.84 \\
$\mathrm{SC} 2$ & 737.5 & 30.73 & 0.72 \\
$\mathrm{SC} 3$ & 828.6 & 34.14 & 1.02 \\
$\mathrm{SC} 4$ & 839.7 & 36.46 & 1.21 \\
SC5 & 813.8 & 32.17 & 0.86 \\
\hline Average & 777.12 & 32.31 & 0.93 \\
Std. dev. & 73.874 & 3.213 & 0.189 \\
Variation [\%] & 9.51 & 9.95 & 20.37 \\
\hline \hline
\end{tabular}

\section{Bending Tests}

Four-point bending tests were carried out to measure the bending stiffness of single-ply TWF. The test setup, based on reference 16, is shown in figure 15. An advantage of using the 4-point bending configuration is that a uniform curvature forms over the central region, whose length can be measured quite accurately, and so the results can be interpreted with greater accuracy than in 3-point bending.

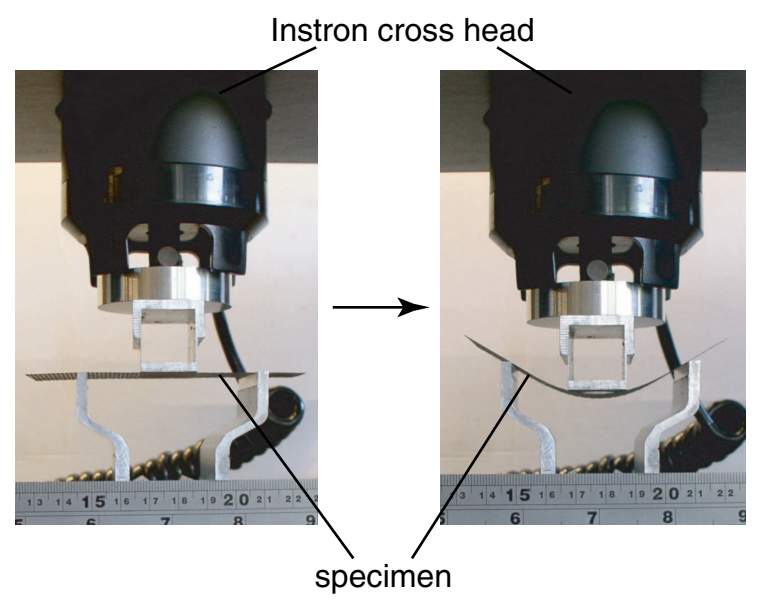

Figure 15. 4-point bending test setup (scale in $\mathrm{mm}$ )

\section{A. Specimens and Test Procedure}

The single-ply specimens were $100 \mathrm{~mm}$ long and $40 \mathrm{~mm}$ wide. The distance between the outer supports was $60 \mathrm{~mm}$ and the distance between the inner supports $20 \mathrm{~mm}$. The deflection of the centre of the specimen with respect to the inner supports was measured by attaching a retro-reflective strip on a side of the specimen and an another strip was attached to the loading head; the relative displacement was measured with an Epsilon LE-05 laser extensometer.

Five tests were performed using an Instron 5578 machine with a $100 \mathrm{~N}$ load cell. A loading rate of 
$1 \mathrm{~mm} / \mathrm{min}$ was applied. Note that in this test only the initial bending stiffness was investigated; the specimens were not taken to failure.

\section{B. Results}

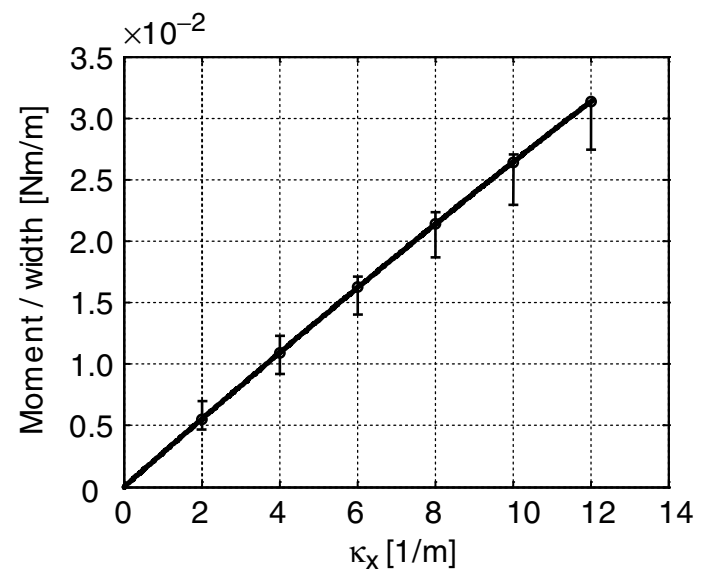

Figure 16. Measured moment per unit width vs curvature

A plot of bending moment per unit width vs longitudinal curvature is shown in figure 16. The relationship is practically linear, and hence the bending stiffness can be estimated. The measured bending stiffnesses are summarized in table 6 . The variation between the 5 tests was only $2 \%$.

Table 6. Measured bending stiffness

\begin{tabular}{cc}
\hline \hline Specimen & bending stiffness, $D_{x}[\mathrm{Nmm}]$ \\
\hline B01 & 2.008 \\
B02 & 2.046 \\
B03 & 2.092 \\
B04 & 2.108 \\
B05 & 2.132 \\
\hline Average & 2.077 \\
Std. dev. & 0.050 \\
Variation [\%] & 2.40 \\
\hline \hline
\end{tabular}

\section{Comparison of Theory and Experiment}

To compare the theoretical results with the experimental measurements it is best to work with the inverse of equation (7). This is because in the experiments we measured the deformations due to unit loads. Hence we define the following $6 \times 6$ compliance matrix 


$$
\left\{\begin{array}{c}
\varepsilon_{x} \\
\varepsilon_{y} \\
\varepsilon_{x y} \\
\kappa_{x} \\
\kappa_{y} \\
\kappa_{x y}
\end{array}\right\}=\left[\begin{array}{ccc|ccc}
a_{11} & a_{12} & a_{16} & b_{11} & b_{12} & b_{16} \\
a_{21} & a_{22} & a_{26} & b_{21} & b_{22} & b_{26} \\
a_{61} & a_{62} & a_{66} & b_{61} & b_{62} & b_{66} \\
\hline b_{11} & b_{21} & b_{61} & d_{11} & d_{12} & d_{16} \\
b_{12} & b_{22} & b_{62} & d_{21} & d_{22} & d_{26} \\
b_{16} & b_{26} & b_{66} & d_{61} & d_{62} & d_{66}
\end{array}\right]\left\{\begin{array}{c}
N_{x} \\
N_{y} \\
N_{x y} \\
\hline M_{x} \\
M_{y} \\
M_{x y}
\end{array}\right\}
$$

The compliance matrix, denoted as abd, is the inverse of the ABD matrix, and is also symmetric $\left(a_{i j}=a_{j i}\right.$ and $\left.d_{i j}=d_{j i}\right)$. Computing the inverse of the matrix in equation (25)

$$
\left\{\begin{array}{c}
\varepsilon_{x} \\
\varepsilon_{y} \\
\hline \kappa_{x} \\
\kappa_{y} \\
\kappa_{x y}
\end{array}\right\}=10^{-6} \times\left[\begin{array}{ccc|ccc}
459 & -276 & 0 & 0 & 0 & 600 \\
-276 & 459 & 0 & 0 & 0 & -600 \\
0 & 0 & 1471 & -600 & 600 & 0 \\
\hline 0 & 0 & -600 & 499220 & -138210 & 0 \\
0 & 0 & 600 & -138210 & 499220 & 0 \\
600 & -600 & 0 & 0 & 0 & 1283000
\end{array}\right]\left\{\begin{array}{c}
N_{x} \\
N_{y} \\
N_{x y} \\
\frac{M_{x}}{M_{y}} \\
M_{x y}
\end{array}\right\}
$$

Consider the case where a tensile load is applied in the $x$-direction

$$
N_{x} \neq 0 \text { and } N_{y}=N_{x y}=M_{x}=M_{y}=M_{x y}=0
$$

Then substitute these values into equation (28). The first row gives

$$
\varepsilon_{x}=a_{11} N_{x}
$$

from which we can predict the extensional stiffness in the $x$-direction

$$
S_{x}=\frac{N_{x}}{\varepsilon_{x}}=\frac{1}{a_{11}}
$$

Considering also the second row of equation (28) and rearranging we can determine the Poisson's ratio from

$$
\nu_{x y}=-\frac{a_{21}}{a_{11}}
$$

Similarly, the shear stiffness can be predicted from

$$
S_{x y}=\frac{N_{x y}}{\varepsilon_{x y}}=\frac{1}{a_{66}}
$$

and finally, the bending stiffness is given by

$$
D_{x}=\frac{M_{x}}{\kappa_{x}}=\frac{1}{d_{11}}
$$

The computed and measured stiffnesses and Poisson's ratio are compared in table 7, where it should be noted that all experimental values correspont to the initial average stiffnesses. Also note that the experimental extensional stiffness has been obtained by averaging the tensile and compressive values. Overall, our predictions agree extremely well with the measurements. 
Table 7. Predicted and measured results

\begin{tabular}{lcc}
\hline \hline Property & Prediction & Measurement (average) \\
\hline Extensional stiffness, $S_{x}[\mathrm{~N} / \mathrm{mm}]$ & 2178 & 2195 \\
Poisson's ratio, $\nu_{x y}$ & 0.601 & 0.586 \\
Shear stiffness, $S_{x y}[\mathrm{~N} / \mathrm{mm}]$ & 680 & 777 \\
Bending stiffness, $D_{x}[\mathrm{Nmm}]$ & 2.003 & 2.077 \\
\hline \hline
\end{tabular}

\section{Discussion}

We have shown that a simple curved beam model subject to periodic boundary conditions predicts very accurately the main stiffnesses of carbon-fibre TWF. The analysis can be easily set up and runs very quickly. Our results are in agreement with Aoki and Yoshida, ${ }^{2}$ although is should be noted that the entries of our ABD matrix are about $50 \%$ larger because we have used a stiffer matrix and a higher fibre volume fraction.

The PBC model presented here only applies to infinitely large pieces of TWF. In finite-size pieces edge effects can be of considerable importance; they have been considered elsewhere. ${ }^{2,3}$ Finally, it should be noted that only linear-elastic behaviour has been considered in this paper; there is obvious scope for extending this work into a geometrically non-linear regime.

\section{Acknowledgments}

We thank Professor T. Aoki and Dr V. Deshpande for helpful discussions. We are grateful to Mr R. Sakai (Sakase Adtech, Japan) and Mr J. Ellis (Hexcel, UK) for providing us with materials and advice on processing techniques. This research was partially supported by ESA-ESTEC. AK thanks Universiti Teknologi Malaysia for the provision of study leave and financial support.

\section{References}

\footnotetext{
${ }^{1}$ Tan, L.T., and Pellegrino, S., Thin-shell deployable reflectors with collapsible stiffeners: Part 1 Approach, AIAA Journal, 44, 2515-2523, 2006.

${ }^{2}$ Aoki, T., and Yoshida, K., Mechanical and thermal behaviors of triaxially-woven carbon/epoxy fabric composite. 47th AIAA/ASME/ASCE/AHS/ASC Structures, Structural Dynamics and Materials Conference, 1-4 May 2006, Newport, RI, 2006.

${ }^{3}$ Kueh, A., Soykasap, O., and Pellegrino, S., Thermo-mechanical behaviour of single-ply triaxial weave carbon fibre reinforced plastic. European Conference on Spacecraft Structures, Materials and Testing (editors: Rocks, J. and Fletcher, K.), 9-13 May 2005, Noordwijk, The Netherlands, ESA SP-581.

${ }^{4}$ Kueh, A., and Pellegrino, S., Thermo-elastic behaviour of single ply triaxial woven fabric composites. 47th AIAA/ASME/ASCE/AHS/ASC Structures, Structural Dynamics and Materials Conference, 1-4 May 2006, Newport, RI, 2006.

${ }^{5}$ Hexcel Composites. Product data sheet, HexPly 8552 epoxy matrix $\left(180{ }^{\circ} \mathrm{C} / 356{ }^{\circ} \mathrm{F}\right.$ curing matrix $), 2003$.

${ }^{6}$ Torayca. Technical Data Sheet No. CFA-001, T300 Data Sheet.

${ }^{7}$ Bowles, D.E., Micromechanics analysis of space simulated thermal deformations and stresses in continuous fiber reinforced composites. NASA Technical Memorandum 102633, 1990.

${ }^{8}$ Daniel I.M., Ishai O., Engineering Mechanics of Composite Materials, second edition, Oxford University Press, 2006.

${ }^{9}$ Whitney J.M., Daniel I.M., Pipes R.B., Experimental Mechanics of Reinforced Composite Materials, Society for Experimental Mechanics, 1984.

${ }^{10}$ Tang, X. and Whitcomb, J.D., General techniques for exploiting periodicity and symmetries in micromechanics analysis of textile composites, Journal of Composite Materials, 37, 13, 1167-1189, 2003.

${ }^{11}$ Karkainen, R.L. and Sankar, B., A direct micromechanics methods for analysis of failure initiation of plain weave textile composites, Composites Science and Technology, 66, 137-150, 2006.

${ }^{12}$ ASTM, Standard Test Method for Tensile Properties of Polymer Matrix Composite Materials, D3039/D3039M-00, United States, 2000.

${ }^{13}$ Fleck, N.A. and Sridhar, I. End compression of sandwich columns. Composites: Part A, 33, 353-359, 2002.

${ }^{14}$ ASTM Standard Test Method for In-Plane Shear Properties of Polymer Matrix Composite Materials by the Rail Shear Method, D4255/D4255M-01, United States, 2001.

${ }^{15}$ Adams, D.F., Carlsson, L.A. and Pipes, R.B., Experimental characterization of advanced composite materials, Third edition, CRC Press, Boca Raton, 2003.

${ }^{16}$ ASTM, Standard Test Method for Flexural Properties of Unreinforced and Reinforced Plastics and Electrical Insulating Materials, D790M-86, United States, 1986.
} 